\title{
U tilidad del estudio molecular de CYP21A2 en el manejo prenatal de hiperplasia suprarrenal congénita: detección de dos nuevas mutaciones en Chile
}

\author{
Paulina Merino1a, Tania Bachega ${ }^{2}$, Pablo Céspedes $^{3}$, León \\ Trejo $^{3}$, Ana Elisa Billerbeck ${ }^{2}$, Ethel Codner ${ }^{3}$. \\ Molecular study of CYP21A2 gene \\ for prenatal diagnosis of congenital \\ adrenal hyperplasia. Report of a \\ family
}

Prenatal treatment of pregnancies at risk of congenital adrenal hyperplasia (CAH) may prevent ambiguous genitalia in female fetuses. We present the prenatal treatment performed in an extended family with two mutations. The proband, a boy with CAH-salt losing form, and his relatives were studied. The proband's paternal uncles/aunts were married to the maternal aunts/uncles, respectively. The relatives had normal basal and stimulated 17OHProgesterone levels, which did not clarify their carrier status. The CYP21A2 gene was sequenced. The proband and the paternal relatives harbored a Q318X, R483W mutation in one allele. The maternal relatives and the proband exhibited an R483 frameshift mutation. Early dexametasone treatment was given during two pregnancies and stopped when male gender was confirmed by early ultrasonography. Both newborns were healthy and had normal 17OHProgesterone levels. This family had three mutations which abolish the 21-hydroxilase activity. Two mutations were detected in codon 483 of CYP21A2 gene, exon 10, which have not been reported previously in Latin-America. The molecular study performed in this family allowed us to give an appropriate genetic counseling and prenatal treatment (Rev Méd Chile 2007; 135: 1450-55).

(Key words: Congenital adrenal hyperplasia; CYP21A2; Progesterone)

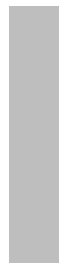
Recibido el 22 de noviembre, 2006. Aceptado el 7 de marzo, 2007.
Trabajo financiado parcialmente por el proyecto Fondecyt 1050452
${ }^{1}$ Departamento de Obstetricia y Ginecología. Escuela de Medicina, Pontificia Universidad Católica de Chile. Santiago, Chile. ${ }^{2}$ Unidad de Endocrinología del Desenvolvimiento, Laboratório de Hormônios e Genética Molecular, LIM 42, Hospital das Clínicas, Facultad de Medicina de Universidad de São Paulo, São Paulo, Brasil. ${ }^{3}$ Instituto de Investigación Materno Infantil (I.D.I.M.I.). Escuela de Medicina, Universidad de Chile. Santiago, Chile.
${ }^{a}$ Médico becado Programa de Especialidad Obstetricia y Ginecología PUC.

T a hiperplasia suprarrenal congénita (HSC) coLrresponde a un grupo de trastornos autosómi-

Correspondencia a: Dra. Ethel Codner. Instituto de Investigación Materno Infantil (I.D.I.M.I.). Escuela de Medicina, Universidad de Chile, Casilla 226-3. Santiago, Chile. Tel: 5624248280. Fax: 562-4247240. E mail: ecodner@med.uchile.cl cos recesivos que afectan las enzimas que participan en la biosíntesis del colesterol ${ }^{1}$. La alteración más frecuente corresponde a la deficiencia de 21hidroxilasa, la que es producida por mutaciones en el gen CYP21A2. Estas mutaciones se han correlacionado con diferentes grados de deficiencia enzimática in vitro y de este modo con la 
gravedad de la presentación clínica, permitiendo que el estudio molecular tenga utilidad en el manejo clínico ${ }^{2}$.

Esta enfermedad, en su variante clásica, produce diferentes grados de virilización del feto femenino, acarreando con ello la necesidad de realizar cirugías de corrección posteriores ${ }^{3}$, y secundariamente consecuencias en la esfera psicológica y en la función sexual de estas pacientes ${ }^{4}$. El tratamiento prenatal con dexametasona logra evitar o reducir el grado de virilización de las niñas afectadas, disminuyendo la necesidad del tratamiento quirúrgico y sus consecuencias $^{5,6}$. El éxito del tratamiento depende en gran parte del inicio precoz de la corticoterapia, logrando mejores resultados cuando se inicia antes de las 7 semanas de gestación. El tratamiento prenatal debe ser mantenido hasta conocer el genotipo fetal, ya que reciben tratamiento en forma innecesaria 7 de 8 embarazos, correspondientes a los fetos de sexo masculino y aquellos fetos femeninos que no están afectados. Con el fin de poder suspender el medicamento en las pacientes que no se beneficiarán de éste, es necesario un diagnóstico precoz de sexo fetal y posteriormente del defecto molecular.

Presentamos el caso de una familia con 3 matrimonios portadores de mutaciones del gen CYP21A2, que comprometen severamente la actividad enzimática y que no habían sido reportadas previamente en Latinoamérica. Se describe el manejo antenatal, desde el punto de vista clínico y molecular en dos embarazos, y se plantean nuevas estrategias diagnósticas.

\section{Caso Clínico}

Familia afectada (Figura 1A). El probando (sujeto III.2) es portador de una HSC perdedora de sal diagnosticada a los 21 días de vida. El estudio hormonal se muestra en la Figura 1B. El paciente ha sido tratado con cortisol y fludrocortisona, y evoluciona en forma satisfactoria con crecimiento y desarrollo normal. No existía el antecedente familiar de HSC, pero destacaba que dos hermanos(as) del padre estaban casados con dos hermanas(os) de la madre.

Estudio molecular y hormonal. El estudio hormonal de la tía y de la madre del probando (sujetos II.2 y II.3) incluyó una prueba de ACTH, cuyos valores de $17 \mathrm{OH}$ Progesterona basal y estimulado fueron normales (Figura 1B), no permitiendo aclarar si estos sujetos eran heterocigotos de una mutación del CYP21A2.

El estudio molecular se realizó en ADN genómico extraído de leucocitos y las siete mutaciones más frecuentes del gen CYP21A2 fueron estudiadas con PCR alelo específica (Fardella, 1998). Este estudio demostró en la familia paterna (sujetos II.2, II.4, II.6) la mutación Q318X en el exón 8, que consiste en el cambio de una glutamina por un codón de stop. Este resultado no permitió clasificar a la familia materna y por ende decidir sobre la necesidad del tratamiento prenatal en estas parejas.

Posteriormente, el gen CYP21A2 fue secuenciado en la Universidad de Sao Paulo según las técnicas clásicas (Wedell, 1993). Usando esta metodología, se encontró en uno de los alelos de la familia paterna (sujetos II.2, II.4, II.6) la mutación Q318X junto a la mutación R483W, la que consiste en la sustitución de una arginina por triptofano (CGG->TGG). En la familia materna (sujetos II.1, II.3, II.5) se encontró un alelo con la mutación R483->frameshift, también conocida como R483delGGinsC, la cuál consiste en el cambio de los nucleótidos GG por C, alterando la lectura del codón (Figura 1C). De este modo se certificó que los seis cónyuges poseen mutaciones para HSC perdedora de sal, y por lo tanto, se encuentran en riesgo de tener hijas con ambigüedad genital.

Consejo genético y manejo de los embarazos. Se procede a dar consejo genético a los tres matrimonios afectados. La tía paterna (sujeto II.2), mujer sana de 34 años, suspende el método anticonceptivo por deseos de fertilidad y logra concepción espontánea en marzo 2005. Se explican los beneficios y riesgos del tratamiento prenatal con corticoides, los que la paciente entiende y acepta, firmando un consentimiento en que opta por realizar esta terapia. El tratamiento comenzó a las 6 semanas de edad gestacional (EG) con dexametasona en dosis de $20 \mu \mathrm{g} / \mathrm{kg} /$ día.

A las 13 semanas se realizó el diagnóstico de sexo fetal masculino por ultrasonografía de alta resolución (Voluson 730, General Electric, Austria, transductor transabdominal de 3,5-5 MHz), la cual es capaz de predecir correctamente el sexo fetal con 99\% de certeza después de 12+6 semanas EG (Efrat, 2006); la familia no aceptó técnicas invasivas de diagnóstico prenatal. Se disminuyó progresivamente las dosis de esteroides, suspendiéndolo en forma 


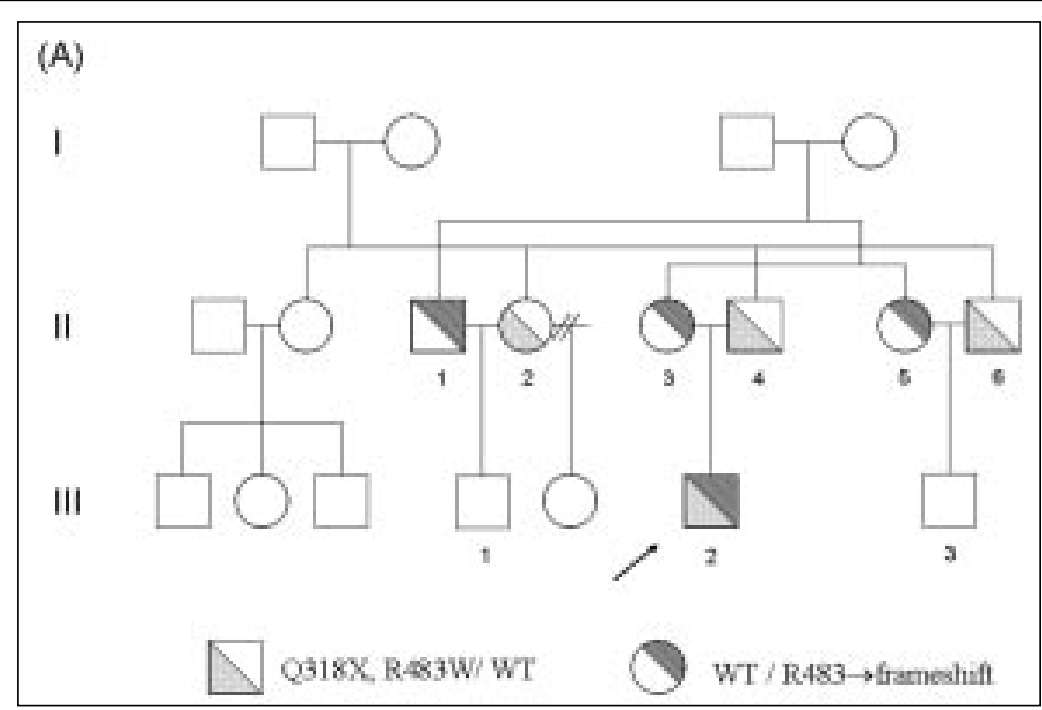

(B)

\begin{tabular}{|llccc|}
\hline & & \multicolumn{2}{c|}{17 OH Progesterona (ng/ } \\
ml) & Sujeto & Genotipo & Edad & Basal Test ACTH \\
\hline III.2 & Q318X, R483W/ R483 $\rightarrow$ frameshift & 22 días & $>20$ & \\
II.2 & Q318X, R483W/ WT & 33 años & 0,5 & 3,6 \\
II.3 & WT / R483 $\rightarrow$ frameshift & 36 años & 0,7 & 3,3 \\
III.1 & Desconocido & 5 días & 0,8 & \\
III.3 & Desconocido & 5 días & 1,1 & \\
\hline
\end{tabular}

(C)

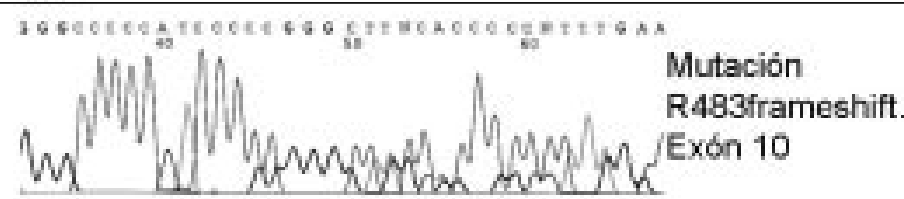

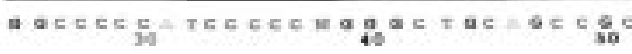

Mutación R483W.

Exón 10

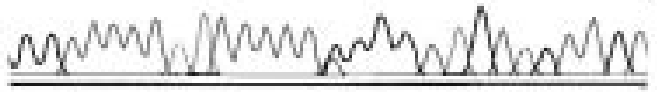

Figura 1. Familia estudio. (A) Genealogía. El probando, sujeto III.2, es indicado por una flecha. WT denota alelo normal. (B) Estudio hormonal de la familia estudiada. (C) Secuenciación del gen CYP21A2 que demuestra las dos nuevas mutaciones detectadas: R483— > frameshift y R483W.

completa a las 22 semanas. Durante el período de tratamiento la paciente presentó edema e hipertensión arterial que requirió tratamiento con ametildopa. Los síntomas revirtieron parcialmente con la suspensión de terapia corticoidal, y totalmente posparto. A las 39 semanas se realizó cesárea y se obtuvo recién nacido de sexo masculino, con peso adecuado y examen físico normal. Los niveles de $17 \mathrm{OHP}$ a los 5 días se muestran en la Figura 1B.

La tía materna (sujeto II.5) de 37 años consultó por embarazo inicial de 5 semanas de EG. Se ofrece nuevamente tratamiento prenatal de HSC, y también se solicitó firma de consentimiento informado. La familia optó nuevamente por seguir este tratamiento. 
A las 6 semanas de EG se inició dexametasona en dosis de $20 \mu \mathrm{g} / \mathrm{kg} /$ día. La madre no aceptó las técnicas invasivas de diagnóstico prenatal y se decidió realizar diagnóstico de sexo fetal mediante ecografía precoz. En caso de que se tratara de un feto femenino continuarían el tratamiento hasta el término del embarazo. A las 12 semanas se diagnosticó sexo fetal masculino y se programó suspensión de esteroides. La paciente no presentó efectos adversos a la dexametasona. A las 40 semanas nació niño sano, no afectado (Figura 1B).

\section{DisCUSIÓN}

Presentamos el caso de una familia con tres matrimonios portadores de mutaciones del gen CYP21A2 que producen HSC perdedora de sal. La secuenciación del gen detectó una mutación en el exón 8 y dos nuevas mutaciones en el exón 10. El estudio molecular permitió tomar la decisión de realizar tratamiento prenatal, hecho que no habría sido posible en base al estudio hormonal, ya que éste resultó normal.

El año 1998, Fardella et al, describió las siete principales mutaciones asociadas a HSC perdedora de sal en pacientes chilenos mediante la técnica de PCR alelo específica ${ }^{7}$. Este estudio concluyó que seis mutaciones producen el $77 \%$ de los casos, con $23 \%$ de falsos negativos. Dado que esta técnica no identificó uno de los alelos afectados, con lo que no era posible realizar consejo genético en los tres matrimonios involucrados, se realizó estudio de secuenciación que reveló las mutaciones R483W y R483 - frameshift, que no se habían detectado en población latinoamericana previamente $\mathrm{e}^{7,10-12}$. La primera fue descrita por primera vez en 2004 en 1 de 51 pacientes con HSC clásica ${ }^{13}$ y la segunda se describió por primera vez en $1993^{8}$.

Una vez conocido el estado heterocigoto de una mutación asociada a la forma clásica, el médico se enfrenta al manejo del embarazo con riesgo de la enfermedad. El sustrato fisiopatológico del tratamiento prenatal se basa en la administración de un esteroide que atraviese la barrera hematoplacentaria y que sea capaz de suprimir el eje hipotálamo-hipófisisadrenal del feto en el momento de formación de los genitales femeninos. Recientemente, Gotto et al demostraron que a las 7 semanas de gestación el eje hipotálamo-hipófisis-suprarrenal está activo y que la secreción de ACTH se suprime ante la administración de dexametasona ${ }^{14,15}$. La literatura ha concluido que los beneficios de la terapia prenatal superan los riesgos, pero debido a la falta de seguimientos a largo plazo, este tipo de tratamiento se debe realizar en centros terciarios, con obtención de consentimiento y seguimiento a largo plazo ${ }^{16}$. El manejo prenatal de los embarazos en esta familia siguen las recomendaciones internacionales (Tabla 1 ).

La principal controversia del tratamiento consiste en la necesidad de tratar 7 de 8 embarazos innecesariamente y en la presencia de efectos adversos en la madre como se observó en uno de los embarazos reportados. Con el fin de poder suspender el tratamiento en aquellos fetos de sexo masculino, surge la necesidad del diagnóstico del sexo fetal. En los embarazos aquí publicados se utilizó la ultrasonografía precoz como método no invasivo de diagnóstico. Este examen busca evaluar la posición del tubérculo genital entre las 11-14 semanas de EG. La sensibilidad de este examen alcanza 97-99\% a las 13 semanas con un alto valor predictivo positivo9,21. Si la adherencia al tratamiento ha sido mala y el feto se ha virilizado, la capacidad de discriminación entre sexo masculino y femenino es baja, por lo que se deben asociar otras técnicas diagnósticas.

En 1997, Lo descubre que el 3\%-6\% del ADN obtenido en sangre de mujeres embarazadas es de origen fetal, lo que permitiría la detección de fetos masculinos en plasma materno ${ }^{22}$. La determinación del sexo mediante el análisis de secuencias $\mathrm{Y}$, tiene una sensibilidad de $95 \%$ antes de las 7 semanas y de 100\% después, para detectar fetos masculinos con $100 \%$ de especificidad y con $2,8 \%-10 \%$ de falsos negativos ${ }^{23,24}$. Estudios recientes demostrarían un rápido eliminación del $\mathrm{ADN}$ tras el parto, por lo que constituye una herramienta útil en futuros embarazos $^{25,26}$. Si bien es promisorio, se requieren más estudios que confirmen el éxito de los resultados ${ }^{27}$.

En el caso de tratarse de un embarazo de sexo femenino se recomienda evaluar el genotipo fetal, por biopsia de vellosidades coriales o amniocentesis, para determinar si es necesario continuar el tratamiento con dexametasona hasta el final del embarazo. Desgraciadamente, ambos procedimientos pueden tener complicaciones, tales como pérdidas fetales o deformidades musculoesqueléticas ${ }^{28,29}$.

En resumen, presentamos una familia en que el estudio molecular por secuenciación detectó nuevas mutaciones que no habían sido descritas en población chilena, y permitió ofrecer consejo genético y 


\section{Tabla 1. Recomendaciones de manejo prenatal}

Indicaciones de tratamiento:

- Padres heterocigotos para mutación causante de HSC clásica.

- Disponibilidad de diagnóstico de sexo fetal y de mutaciones de CYP21A2.

- Edad gestacional menor a 7 semanas.

Medicamento de elección: Dexametasona

- Dosis: $20 \mathrm{ug} / \mathrm{kg} /$ día, fraccionada cada 8 horas.

- Inicio: antes de las 6 semanas de gestación.

- Término tratamiento con dexametasona:

- Si sexo fetal es masculino se suspende de inmediato.

- Si sexo es femenino, se realiza el estudio molecular del gen CYP21A2. Si feto no está afectado, la dexametasona se suspende de inmediato, por el contrario si feto es homocigoto, se mantiene el tratamiento hasta el parto, disminuyendo la dosis desde la semana 16.

Evaluación de sexo fetal y genotipo CYP21A2:

- Primera etapa: determinación de sexo fetal (ADN libre fetal, ultrasonido precoz).

- Segunda etapa: caracterización de defecto molecular mediante técnicas invasivas (amniocentesis, biopsia de vellosidades coriales) a los fetos femeninos.

Efectos benéficos:

- Reducción del score de Prader en $85 \%$ de las pacientes tratadas, las que presentan genitales femeninos normales o con leve virilización cuando el tratamiento se instaura precozmente y se realiza en forma completa. Las fallas en el tratamiento se deben a un inicio tardío o una suspensión temprana de los esteroides, no adherencia al tratamiento, uso de dosis subóptima y diferencias en el metabolismo placentario de la dexametasona ${ }^{6}$.

Efectos adversos:

- Sobre el niño: asociación dudosa con bajo peso de nacimiento. Estudios en animales sugieren alteraciones de la sustancia blanca e hipocampo. Datos recientes sugieren deterioro de la memoria verbal y en la autopercepción derivada de éste déficit. Pendiente estudios a largo plazo $^{17-19}$.

- Sobre la madre: 10\%-30\% de las pacientes presentan efectos adversos, entre los cuales están el edema, la hipertensión, el síndrome de Cushing y el aumento de las complicaciones de comorbilidades previas como diabetes e HTA ${ }^{20}$.

tratamiento prenatal. Esperamos que en los próximos años podamos contar con diagnóstico de sexo y genotipo fetal en sangre materna con el fin de minimizar los riesgos asociados al proceso diagnóstico y terapéutico.

\section{REFERENCIAS}

1. Speiser PW, White PC. Congenital adrenal hyperplasia. N Engl J Med 2003; 349: 776-88.

2. Robins T, Carlsson J, Sunnerhagen M, Wedell A, Persson B. Molecular model of human CYP21 based on mammalian CYP2C5: Structural features correlate with clinical severity of mutations causing congenital adrenal hyperplasia. Mol Endocrinol. 2006; 20: 2946-64.

\section{Agradecimientos}

Agradecemos al Dr. Hernán Muñoz, Clínica Las Condes, Santiago, Chile, por la realización de las ecografías de alta resolución.

3. Farkas A, Chertin B, Hadas-Halpern I. 1-Stage feminizing genitoplasty: 8 years of experience with 49 cases. J Urol 2001; 165: 2341-6.

4. Crouch NS, Minto Cl, Laio LM, Woodhouse CRJ, Creighton SM. Genital sensation after feminizing genitoplasty for congenital adrenal hyperplasia: a pilot study. BJU Int 2004; 93: 135-8.

5. Ritzen EM, LajIC S. Outcome of Prenatal Dexamethasone Treatment of Congenital Adrenal Hyperplasia. The Endocrinologist 2003; 13: 219-23. 
6. New M, Carlson A, Obeid J, Marshall i, Cabrera M, Goseco A, ET AL. Update: Prenatal Diagnosis for Congenital Adrenal Hyperplasia in 595 Pregnancies. The Endocrinologist 2003; 13: 233-9.

7. Fardella C, Poggi H, Pineda P, Soto J, Torrealba I, Cattani A et al. Salt-Wasting Congenital Adrenal Hyperplasia: Detection of Mutations in CYP21B Gene in a Chilean Population. J Clin Endocrinol Metab 1998; 83: 3357-60.

8. Wedell A, Luthman H. Steroid 21-hydroxylase (P450c21): a new allele and spread of mutations through the pseudogene. Hum Genet 1993; 91: 23640 .

9. Efrat Z, Perri T, Ramati E, Tugendreich D, Meizner I. Fetal gender assignment by first-trimester ultrasound. Ultrasound Obstet Gynecol 2006; 27: 619-21.

10. Bachega T, Billerbeck Ae, Madureira G, Marcondes J, Longui Ca, Leite Mv et al. Molecular Genotyping in Brazilian Patients with the Classical and Nonclassical Forms of 21-Hydroxylase Deficiency. J Clin Endocrinol Metab 1998; 83: 4416-9.

11. Dardis A, Bergada I, Bergada C, Rivarola M, Belgorosky. Mutations of the steroid 21-hydroxylase gene in an Argentinian population of 36 patients with classical congenital adrenal hyperplasia. J Pediatr Endocrinol Metab 1997; 10: 55-61.

12. Ordoñez-Sánchez ML, Ramírez-Jiménez S, López-GutiérRez Au, Riba L, Gamboa-Cardiel S, Cerrillo-Hinojosa M, ET AL. Molecular genetic analysis of patients carrying steroid 21-hydroxylase deficiency in the Mexican population: identification of possible new mutations and high prevalence of apparent germline mutations. Hum Genet. 1998; 102: 170-7.

13. Kharrat M, Tardy V, Mrad R, Maazoul F, Jemaa Lb, Refaï M eT AL. Molecular Genetic Análisis of Tunisian Patients with a Classic Form of 21-Hydroxylase Deficiency: Identification of Four Novel Mutations and High Prevalence of Q318X Mutation. J Clin Endocrinol Metab 2004; 89: 368-74.

14. Goto M, Hanley KP, Marcos J, Wood PJ, Wright S, POSTLE AD ET AL. In humans, early cortisol biosíntesis provides a mechanism to safeguard female sexual development. J Clin Invest 2006; 116: 953-60.

15. White PC. Ontogeny of adrenal steroid biosynthesis: why girls will be girls. J Clin Invest 2006; 116: 872-4.

16. Joint Lwpes/Espe CaH Working Group. Consensus Statement on 21- Hydroxylase Deficiency from The Lawson Wilkins Pediatric Endocrine Society and The European Society for Paediatric Endocrinology. J Clin Endocrinol Metab 2002; 87: 4048-53.
17. Lajic S, Wedell A, Bui Th, Ritzen Em, Holst M. Long-term somatic follow-up of prenatally Treated Children with Congenital Adrenal Hyperplasia. J Clin Endocrinol Metab 1998; 83: 3872-80.

18. Meyer-Bahlburg HF, Dolezal C, Baker SW, Carlson AD, OBeID JS, New MI. Cognitive and motor development of children with and without congenital adrenal hyperplasia after early-prenatal dexamethasone. J Clin Endocrinol Metab. 2004; 89: 610-4.

19. Hirvikoski T, Nordenström A, Lindholm T, Lindblad F, Ritzén M, Wedell A, Lajic S. Prenatal Dexamethasone Treatment of Children at Risk for Congenital Adrenal Hyperplasia Affects Cognitive Functions. J Clin Endocrinol Metab 2006; en prensa.

20. Forest MG, Dörr HG. Prenatal Therapy in Congenital Adrenal Hyperplasia Due to 21-Hydroxylase Deficiency: Retrospective Follow-Up Study of 253 Treated Pregnancies in 215 Families. The Endocrinologist 2003; 13: 252-9.

21. Ara R, Rodríguez N, Salinas H. Determinación del sexo genérico fetal por ultrasonografía precoz: 1114 semanas. Rev Chil Ultrasonog 2005; 8: 109-13.

22. Lo YM, Corbetta N, Chamberlain PF, Rai V, Sargent IL, Redman CW, Wainscoat JS. Presence of fetal DNA in maternal plasma and serum. Lancet 1997; 350: 485-7.

23. Honda H, Miharu N, Ohashi Y, Samura O, Kinutani M, Hara T et al. Fetal gender determination in early pregnancy through qualitative and quantitative análisis of fetal DNA in maternal serum. Hum Genet 2002; 110: 75-9.

24. Galbiati S, Smid M, Gambini D, Ferrari A, Restagno G, VIORA E ET AL. Fetal DNA detection in maternal plasma throughout gestation. Hum Genet 2005; 117: 243-8.

25. Smid M, Galbiati S, Vasallo A, Gambini D, Ferrari A, VIORA E ET AL. No evidence of fetal DNA persistence in maternal plasma alter pregnancy. Hum Genet 2003; 112: 617-8.

26. Lo YM, Leung TN, Lau TK, Chang AM, Hjelm NM. Rapid clearance of fetal DNA from maternal plasma. Am J Hum Genet 1999; 64: 218-24.

27. Nimkarn S, New M. Prenatal Diagnosis and Treatment of Congenital Adrenal Hyperplasia. Horm Res 2007; 67: 53-60.

28. Philip J, Silver RK, Wilson RD, Thom EA, Zachary JM, Mohide P ET AL. Late First-Trimester Invasive Prenatal Diagnosis: Results of an International Randomized Trial. Obstet Gynecol 2004; 103: 1164-73.

29. Cenerholm M, Haglund B, Axelsson O. Infant morbidity following amniocentesis and chorionic villus sampling for prenatal karyotyping. BJOG 2005; 112: 394-402. 